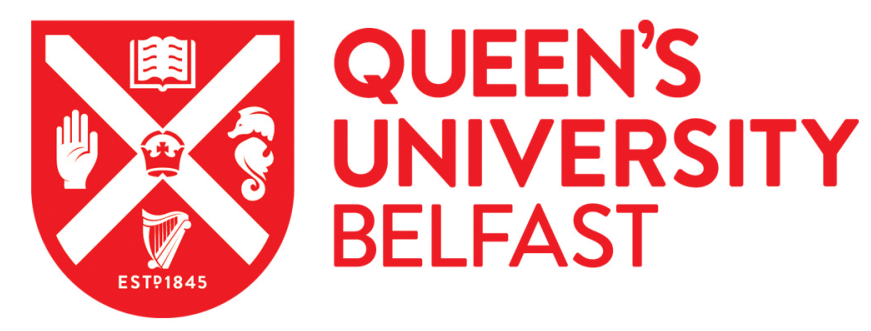

\title{
A one-pot method for building colloidal nanoparticles into bulk dry powders with nanoscale magnetic, plasmonic and catalytic
} functionalities

Ye, Z., Li, C., Skillen, N., Xu, Y., McCabe, H., Kelly, J., Robertson, P., \& Bell, S. (2019). A one-pot method for building colloidal nanoparticles into bulk dry powders with nanoscale magnetic, plasmonic and catalytic functionalities. Applied Materials Today, 15(Special Issue Plasmonics), 398-404.

https://doi.org/10.1016/j.apmt.2019.03.004

Published in:

Applied Materials Today

Document Version:

Publisher's PDF, also known as Version of record

Queen's University Belfast - Research Portal:

Link to publication record in Queen's University Belfast Research Portal

\section{Publisher rights}

Copyright 2019 Elsevier.

This manuscript is distributed under a Creative Commons Attribution-NonCommercial-NoDerivs License

(https://creativecommons.org/licenses/by-nc-nd/4.0/), which permits distribution and reproduction for non-commercial purposes, provided the author and source are cited.

\section{General rights}

Copyright for the publications made accessible via the Queen's University Belfast Research Portal is retained by the author(s) and / or other copyright owners and it is a condition of accessing these publications that users recognise and abide by the legal requirements associated with these rights.

\section{Take down policy}

The Research Portal is Queen's institutional repository that provides access to Queen's research output. Every effort has been made to ensure that content in the Research Portal does not infringe any person's rights, or applicable UK laws. If you discover content in the

Research Portal that you believe breaches copyright or violates any law, please contact openaccess@qub.ac.uk. 


\title{
A One-Pot Method for Building Colloidal Nanoparticles into Bulk Dry Powders with Nanoscale Magnetic, Plasmonic and Catalytic Functionalities
}

Ziwei Ye, Chunchun Li, Nathan Skillen, Yikai Xu*, Hannah McCabe, Jessica Kelly, Peter Robertson, Steven E. J. Bell*

School of Chemistry and Chemical Engineering

Queen's University of Belfast

University Road, Belfast, BT7 1NN, Northern Ireland, United Kingdom

Keywords: self-assembly, hybrid material, SERS, photocatalyst, bulk-nano material

\begin{abstract}
Building easy-to-handle bulk materials with nanoproperties is crucial for many nanotechnology-based real-world applications. Here, we describe a simple onepot method based on nanoparticle self-assembly of pickering emulsions and in-situ polymer deposition for preparing particles consisting of a rigid micro-polymer core covered in exposed surface layers of nanoparticles, which we have named nanomicro-particles (NMPs). Unlike simple colloids, these NMPs can be filtered off from the aqueous suspensions in which they are prepared and dried to form free-flowing powders which, most importantly, retain the properties of the constituent nanoparticles in the surface layer. These NMPs can be stored for extended periods but then used either in the dry state or be re-suspended into liquid media as required. The preparation method is very general and can be readily extended to assemble various types of nanoparticles regardless of their material composition or morphology. In addition, functional components, such as magnetic particles or fluorescent tags, can be encapsulated within the polymer core. This method is a platform technology for building nanoparticles into bulk materials with nano-functionalities tailored towards real-life applications. This is illustrated with examples of the preparation of NMPs suitable for rapid and low-cost on-site water monitoring and remediation.
\end{abstract}

\section{Introduction}

One of the main challenges in nanotechnology is to build nanoscale components into bulk materials which are suitable for real world applications without losing the functionality of their nanoscale constituents. A possible elegant solution to this problem is to start with nanoparticle (NP) building blocks and arrange them into bulk $2 \mathrm{D}$ or $3 \mathrm{D}$ assemblies. These NP assemblies often display intriguing catalytic, magnetic and plasmonic properties that stem from both the properties of the individual NP building blocks and the electron coupling between neighboring NPs [14]. In this work, we present a facile method based on room-temperature self-assembly to fabricate bulk quantities of 3D nanoarrays in the form of solid microparticles with exposed nanoparticle surfaces which are large and strong enough for routine handling but also exhibit useful nanoproperties. Importantly, the method can be readily extended to various different types of NP building blocks regardless of their morphology and material composition, thus adding to the generality and utility of our approach. Here we show examples of different functionality that can be incorporated into these assemblies and their application in a complex real-life system, contaminated river water, where particles which were optimised for on-site tracedetection or for photodecomposition of contaminants in the water were demonstrated. 
3D NP assemblies have been widely investigated due to their potential in a variety of applications such as sensing, drug release and catalysis [5-7]. One way to build 3D structures is by attaching colloidal NPs onto pre-formed micro-particle cores to produce satellite structures [8-11]. In this case, the core particle acts as a hard template during fabrication and provides additional physical robustness to the final 3D structure. However, this method requires multiple sophisticated chemical functionalization steps which often results in a sparse NP loading. Alternatively, densely packed 3D NP arrays such as pickering emulsions and colloidosomes can be fabricated through bottom-up self-assembly by using liquid-liquid interfaces (LLI) as soft-templates [12-15]. While these materials exhibit excellent functionalities they lack the physical and chemical robustness which is crucial for many real-world applications. Therefore, various methods such as thermal annealing, interparticle crosslinking, gel trapping etc. have been proposed to strengthen the structure of such interfacial 3D NP arrays [16-19]. However, these methods either induce significant changes to the structure of the particle layers, which severely compromises their activity, or they require chemical functionalization steps which are inconvenient and material-specific.

Ideally, what is required is a simple method that can be used to arrange various types of NPs into robust 3D materials which are strong enough for routine handling while retaining their nanoproperties. Previously, we have shown that charged colloidal NPs of various morphologies and material compositions can be induced to self-assemble into densely packed 2D arrays at the LLI using oppositely charged promoter ions to provide charge screening [20,21]. In the current work we extend our approach for assembling $2 \mathrm{D}$ arrays to the assembly of densely packed interfacial 3D arrays, this is then combined with in-situ polymer deposition to form suspensions of $\mathrm{NP} /$ polymer hybrid materials consisting of exposed and densely packed NP surface layers fixed on solid polymer micro-particle cores. Since neither the self-assembly nor polymer deposition process involves material-specific chemical reactions, our approach can be readily used to generate robust 3D materials with various types of hydrophilic NP surface layers ranging from metals to oxides and even composites. Moreover, since the polymer core forms at the organic side of the liquid-liquid interface and the NPs partly penetrate into the organic layer, in the product the NPs are firmly anchored onto the polymer core while being both physically and chemically exposed to their surroundings. As a result, our material can be dried into bulk powders which can be easily stored and then dispersed into various complex systems for nanoapplications and then recovered by filtration, centrifugation etc. We have named this new class of nano-powder material - nano-micro-particles (NMPs).

\section{Results and Discussion NMP preparation}

The entire procedure for fabricating NMPs is illustrated in Figure 1. Here, colloidal Au NPs were used for the convenience of illustration but the method can be readily applied to form NMPs from various types of NP building blocks, as shown below. When a mixture of aqueous Au colloid and a highly water immiscible oil such as dichloromethane (DCM) is emulsified to form oil-in-water emulsions, the NPs are spontaneously driven to the interface to lower the energy of the LLI. However, since hydrophilic NPs are inherently charged, the NPs will not accumulate at the LLI (due to the increasing Coulombic repulsion that occurs) unless oppositely charged promoter ions are added to provide charge screening between adjacent particles (Fig. $1 \mathrm{a}$ and $\mathrm{b}$ ). This promoter based approach provides a general method for reducing the 
electrostatic repulsion between the NPs at the interface without the need to perform inconvenient material-specific surface modifications. The processes which occur subsequent to particle localization depend on the experimental conditions, specifically whether there are sufficient particles to cover the whole LLI of the emulsion. If the number of Au NPs is low (Fig. 1b), the initially formed emulsion droplets will be only partly covered with NPs and will thus coalesce to minimize the surface energy by reducing the interfacial surface area, finally forming a smooth reflective 2D film which encloses the coalesced oil phase i.e. a metal liquid-like film (MeLLF), as shown in Figure 1c and d. As the number of particles present increases, a critical point is reached where there are enough NPs to fully cover the coalesced oil phase. If the number of NPs in the system exceeds this critical value, the emulsion droplets become completely covered in particles before they have fully coalesced to form a MeLLF (Fig. 1e and g). However, simply having a high NP surface coverage at the LLI is not enough to stabilize the droplets and stop the emulsions from coalescing. In addition, the NPs need to be sufficiently hydrophilic to stabilize the water meniscus between oil droplets [22]. In our case, this was not a problem since we used aqueous colloids, which meant that most of the NPs were already sufficiently hydrophilic (see Fig. 1 and Table 1 in ref. [23] for detailed mechanism). Under these conditions, the particle covered emulsion droplets remain separated long enough for the dichloromethane (DCM) in the droplet to evaporate. During this evaporation the pre-dissolved polystyrene precipitates at the organic side of the LLI, which leads to the formation of a suspension of rigid polymer microspheres with densely packed NP layers anchored on the surface i.e. NMPs (Fig. 1f). Since the NPs are firmly anchored on the polymer core, they are prevented from aggregating even when dry. This means that the NMPs can be dried into bulk powders, which can be redispersed into solution during application (Fig. 1h). Even on the bench scale, multigramme batches of NMPs can be produced. Fig. $1 \mathrm{~h}$ shows a ca. $0.5 \mathrm{~g}$ batch of NMPs prepared from just $50 \mathrm{~mL}$ of colloidal gold.

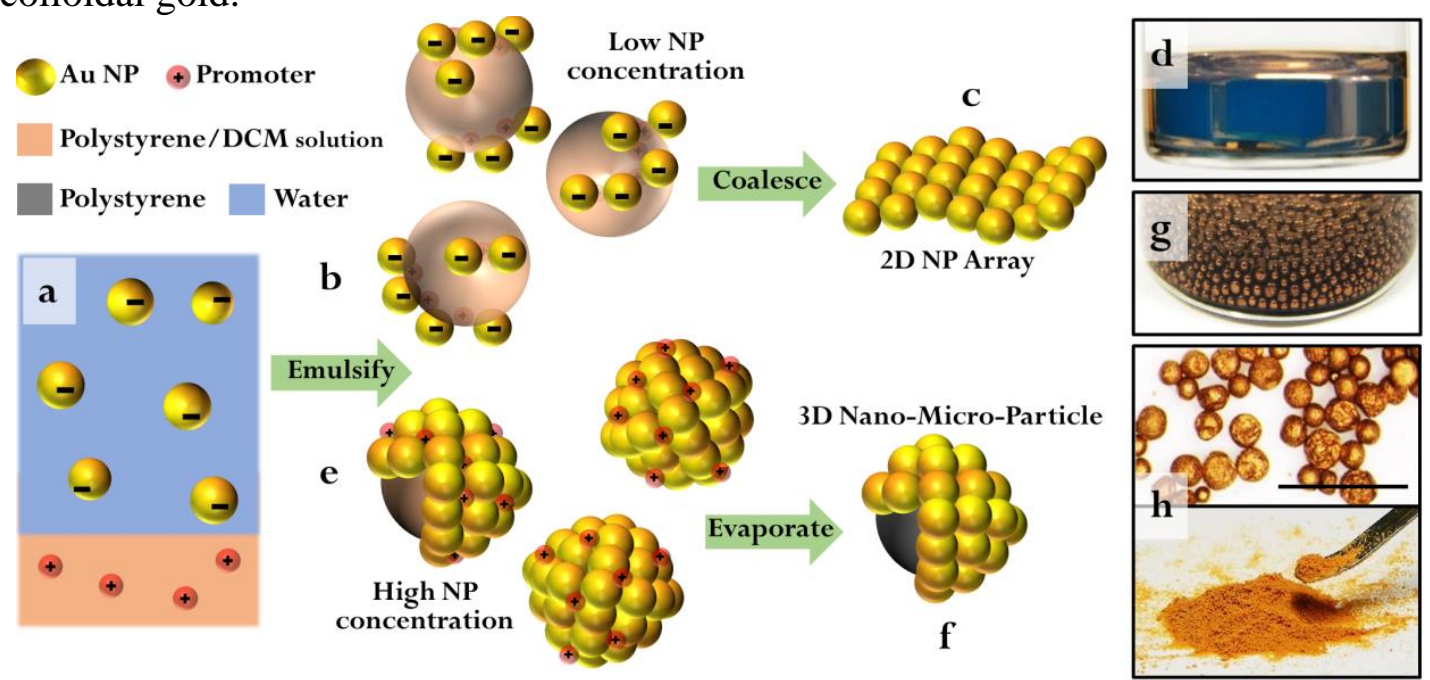

Figure 1. Schematic illustration of Au nanoparticles (NPs) assembling at the liquid-liquid interface (LLI). When a low concentration of NPs is used for selfassembly, the NPs migrate to the LLI during emulsification to form Pickering emulsions, which quickly coalesce to form a smooth reflective MeLLF (a)-(c). Pickering emulsions formed with densely packed hydrophilic NPs remain stable and can therefore be transformed into nano-micro-particles (NMPs) after complete evaporation of dichloromethane (DCM) (e)-(f). Optical images of MeLLFs, Pickering 
emulsions and NMPs formed with Au NPs are shown in (d), (g), (h), respectively. The scale bar in (h) corresponds to $1 \mathrm{~mm}$.

\section{NMP characterization}

The morphology of the NMPs was found to be dependent on the concentration of polystyrene in the oil phase at a given colloid-oil ratio, and fell into the four morphology categories described by Feng [24]. Smooth NMPs were obtained at a higher polystyrene concentration while wrinkled NMPs were more likely to be observed at lower polystyrene concentration (Fig. 2a and b). These morphologies indicate that the particle layers on the outside of the emulsion droplets behave like thin continuum elastic shells during the buckling caused by DCM evaporation [25]. More specifically, the NP array at the LLI is jammed together to form a multilayer particle shell of a certain thickness during the initial stage of oil evaporation. The thickness of the particle shell is set by the point when it becomes robust enough to act as an elastic membrane. Beyond this point, further evaporation of the encapsulated oil phase only causes the shell to distort or buckle, rather than grow thicker. Indeed, SEM images of thin cross-sections (Fig. 2c and d) and surfaces (inset images of Fig. 2a and b) of smooth and wrinkled NMPs show that the particle shells had similar thickness and micro-morphology in both, which is consistent with this elastic shell model. Figure 2a and b show two extreme cases where the morphology of the dried NMP is totally smooth or completely buckled. However, since the buckling of the outer shell is halted when evaporation reaches the point where the polystyrene precipitates out to form the rigid inner core, the morphology can be fixed at any desired stage in between these extremes by altering the concentration of the polystyrene in the original mixture (see Fig. 2 in ref. [23])

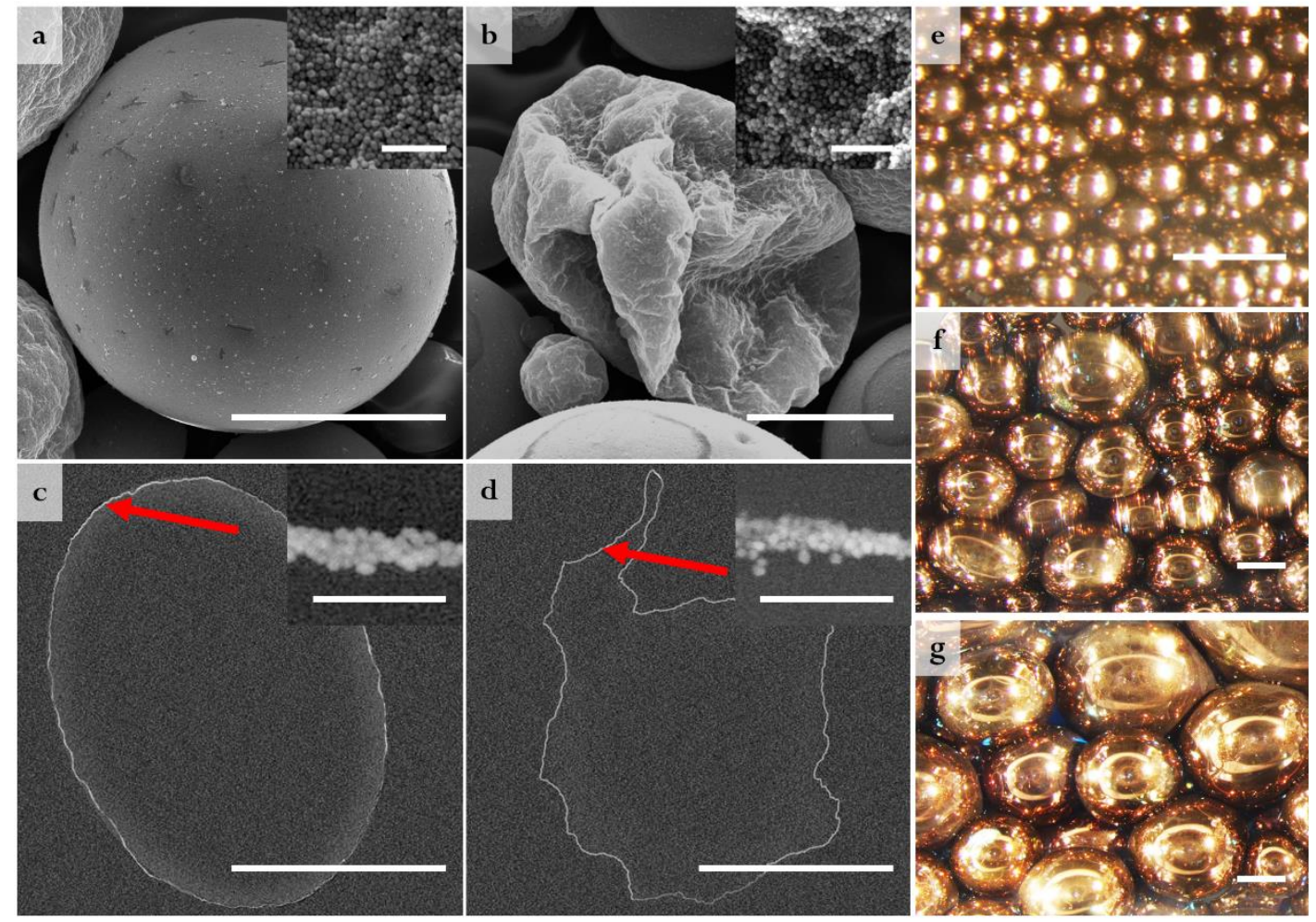

Figure 2. Scanning electron microscopy (SEM) images showing NMPs exhibiting different morphologies: (a) smooth, (b) wrinkled. Insets show high magnification images of the NMPs' surfaces. (c), (d) show SEM images of a thin section of a smooth NMP at low magnification. Insets show their corresponding high 
magnification images. (e)-(g) are optical images of gold emulsions corresponding to different colloid-oil ratios: 5:1, 5:1.5, 5:2 before evaporation. Scale bars in (a)-(d) correspond to $30 \mu \mathrm{m}$. Scale bars in the insets of (a)-(d) correspond to $250 \mathrm{~nm}$. Scale bars in (e)-(g) correspond to $1 \mathrm{~mm}$.

For real world applications it is useful to be able to tailor materials with optimized physical properties. One of the important benefits of our approach is that the size as well as the morphology of the product NMPs can be controlled by changing the droplet size of the parent emulsions. This can be achieved by simply altering the colloid-oil ratios, as shown in Figure 2e-g. Obviously, lower colloid-oil ratio will result in larger emulsion droplets, leading to the formation of larger NMPs and vice-versa. Therefore, NMPs from 10's to 100's of $\mu \mathrm{m}$ in diameter can be prepared this way. The relationship between the product NMP size and the experimental variables can be derived from simple geometrical considerations and is given by Equation 1:

$R=\alpha \times 3.77 \times v \times \sqrt[3]{c} /\left(d^{2} \times n\right)$

where $\mathrm{R}$ is the radius of NMPs; $\alpha$ is a correction factor to compensate for the increased surface area due to wrinkling; $d$ is the diameter of constituent NPs; $v$ is the volume of oil phase; $\mathrm{n}$ is the number of NPs added and $\mathrm{c}$ is the polymer concentration. The derivation of this expression and representative 3D plots showing how the radius of NMPs change with variations in NP-oil ratio and polymer concentration are shown in Figure 4 in ref. [23].

The data above used Au NPs as an example but the promoter-induced selfassembly method is extremely versatile since it allows the assembly of hydrophilic particles at LLIs independent of their morphology and chemical composition. This has allowed us to prepare NMPs from a broad range of NPs while still retaining the key structural features of a rigid polymer core with a densely packed surface layer of exposed particles. For illustration, here we show further examples of NMPs with Ag, $\mathrm{Pt}, \mathrm{TiO}_{2}, \mathrm{SiO}_{2}$ and $\mathrm{Fe}_{3} \mathrm{O}_{4}$ (magnetite) NP surface layers (Fig. 3a-e) but there is no fundamental reason why the approach could not be extended to any type of hydrophilic particle of interest or even mixtures of different particles, such as Ag and $\mathrm{Au}$, as shown in Figure 3f. These examples were chosen to show the same basic approach can be used to create NMPs with very different types of functionality depending on the NPs used as the shell. For example, NMPs with plasmonic absorption are prepared with $\mathrm{Au}$ or $\mathrm{Ag}$, while the $\mathrm{TiO}_{2} \mathrm{NMPs}$ are colorless but photocatalytically active; the magnetite particles have a high magnetic susceptibility and the Pt NMPs are catalytically active. 


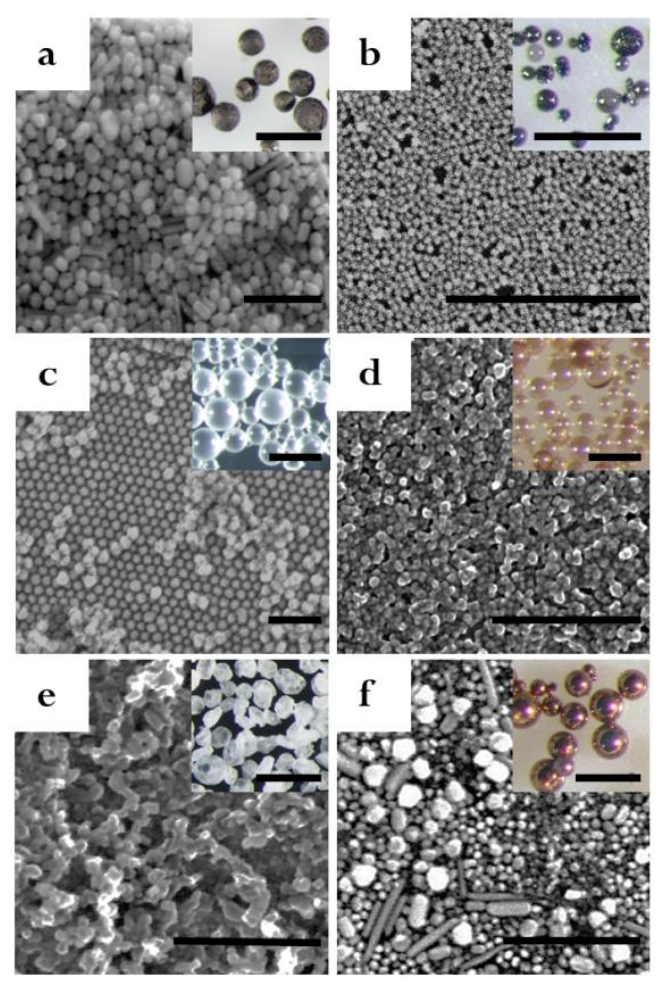

Figure 3. SEM images of the surfaces of NMPs made with various NPs including: (a) $\mathrm{Ag}$, (b) $\mathrm{Pt}$, (c) $\mathrm{SiO}_{2}$, (d) $\mathrm{Fe}_{3} \mathrm{O}_{4}$, (e) $\mathrm{TiO}_{2}$, (f) mixed $\mathrm{Au}$ and $\mathrm{Ag}$. Insets show corresponding optical images of the dry NMPs. Scale bars in SEM and optical images correspond to $500 \mathrm{~nm}$ and $250 \mu \mathrm{m}$, respectively.

\section{Encapsulation of functional materials}

Since the rigid cores of the NMPs are created by solvent evaporation, they can be readily used to encapsulate additional functional components by dispersing them in the oil phase of the stabilized emulsion droplets. To illustrate this aspect of the method, $\mathrm{TiO}_{2}$ covered NMPs were prepared from dichloromethane containing either a fluorescent dye (Rhodamine 6G) or hydrophobic (oleic acid capped) magnetic particles, along with the required concentration of polystyrene. $\mathrm{TiO}_{2}$ particles were used for the surface NP layer because they are colorless and therefore allowed both the incorporation of the brown magnetic particles and the red fluorescence of the dye to be observed optically (Fig. $4 \mathrm{a}$ and b). The magnetic NMPs prepared with encapsulated hydrophobic magnetite NPs could be attracted to the side of a vessel with a permanent magnet. To confirm that the oleic acid capped magnetic particles were indeed located in the core, rather than at the surface (as was the case for the hydrophilic $\mathrm{Fe}_{3} \mathrm{O}_{4}$ NMP shown in Fig. 3d), the NMPs were sonicated for 12 hours to remove all the surface particles (see Fig. 3 in ref. [23]). Subsequent inspection showed that they were still brown and magnetic and therefore that the magnetic particles were located in the core.

\section{NMPs for water monitoring and remediation}

An example which illustrates the versitile functionalities of NMPs is shown in Figure 4, where plasmonic Au NMPs and photocatalytic $\mathrm{TiO}_{2} \mathrm{NMPs}$ were used to both detect and remove organic pollutants in river water. Organic compounds are used in numerous human activities and consequently have become ubiquitous pollutants in natural water resources, which poses a serious threat to the ecosystem even at 
extremely low concentrations. [26,27]. Despite the various methods which have been developed to combat this problem water pollution has remained a global issue [28-30]. Recently, surface-enhanced Raman spectroscopy (SERS) has emerged as a potential candidate for rapid and low cost on-site water monitoring [31-34]. However, the application of SERS to on-site water monitoring has not been straightforward due to a lack of suitable enhancing substrates. Conventional colloidal NP substrates are wellknown to give strong signal enhancements but a combination of difficulty with storage, the need for liquid handling steps and matrix interference with particle aggregation makes them unsuitable for on-site measurements. In contrast, Au NMPs retain the strong signal enhancement of colloidal $\mathrm{Au}$ NPs due to their exposed plasmonic surface, they can be conveniently stored until required and they can be easily dispersed into and recovered from solution like any bulk material. Figure $4 \mathrm{c}$ shows the SERS signals of river water samples spiked with various concentrations of crystal violet $(\mathrm{CV})$ which were obtained using individual magnetic $\mathrm{Au}$ NMPs (i.e. $\mathrm{Au}$ NMPs with magnetite particles in the core). In each case a single magnetic Au NMP was dropped into the samples and agitated for 60 s before being recovered using a permanent magnetic, rinsed with water and analyzed. The signal of CV can be clearly seen even at $10^{-7} \mathrm{M}$, which is below the FDA standard limit for cationic dyes in drinking water [35]. Since the plasmonic particle layer on the surface of NMPs is exposed, this means that they can be pre-functionalized with a SERS standard such as 4-mercaptobenzoic acid (MBA) to normalize signal uniformity between different NMPs and allow accurate and reproducible quantitative analysis, as shown in the inset of Figure 4c. Moreover, the high physical robustness and chemical stability of the NMPs allows them to function even in more complex and challenging sampling environments, such as muddy river water, in which (see Figure S1) CV has been detected $<10^{-6} \mathrm{M}$, exceeding the detection limit of conventional colloidal substrates by several orders of magnitute. 

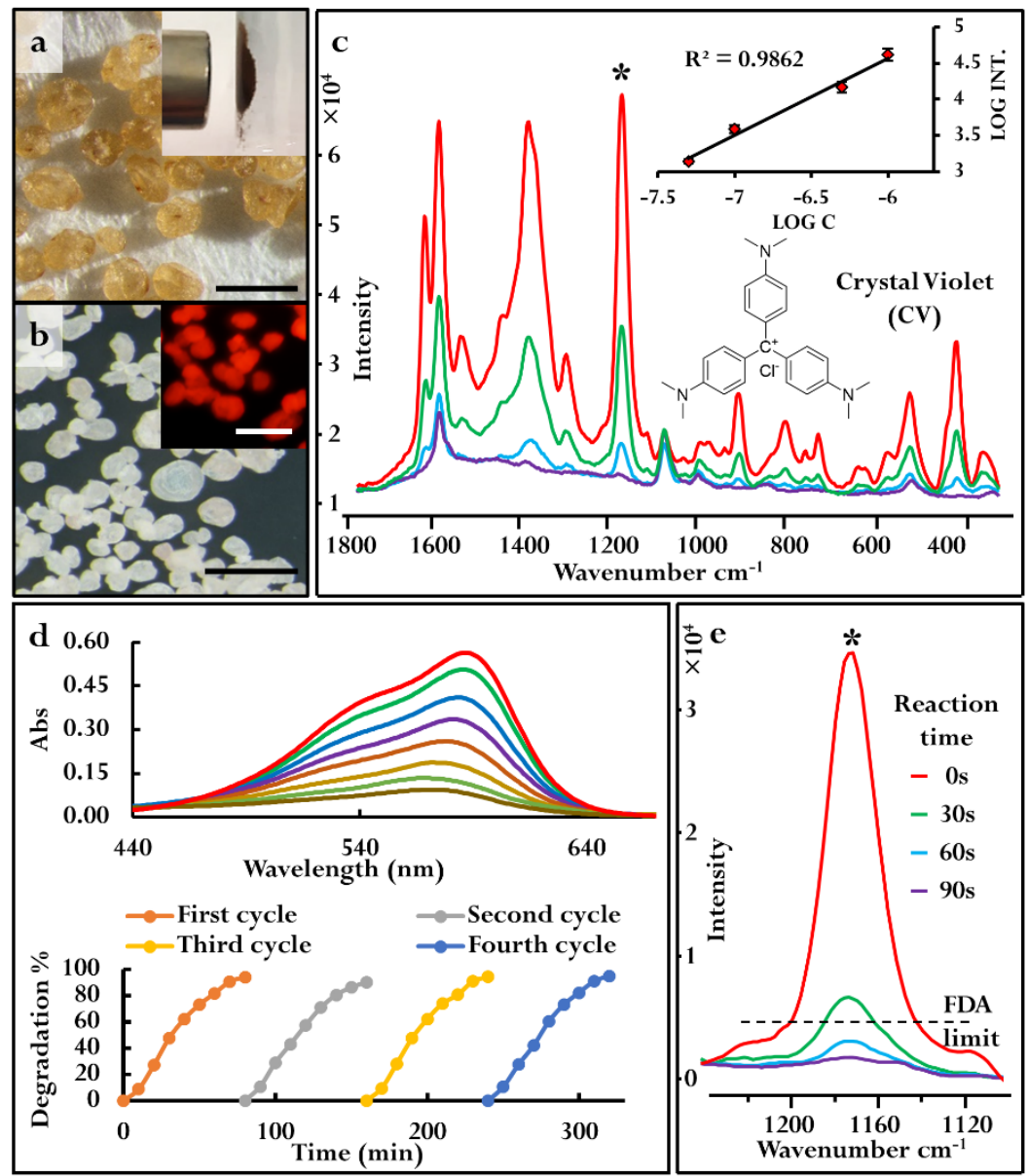

Figure 4. NMPs for water monitoring and remediation. Optical images of $\mathrm{TiO}_{2}$ NMPs with (a) magnetic particles or (b) dye molecules encapsulated in their polymer cores. Inset in (a) shows $\mathrm{TiO}_{2}$ NMPs with a magnetic core being attracted to the wall of the vessel by a magnet. Inset in (b) shows the dye encapsulated $\mathrm{TiO}_{2} \mathrm{NMPs}$ observed using a fluorescence microscope. Scale bars in (a) and (b) correspond to 250 $\mu \mathrm{m}$. (c) SERS spectra of river water samples spiked with crystal violet (CV) and measured using single magnetic Au NMPs capped with 4-mercaptobenzioc acid (MBA) internal standard $\left(1076 \mathrm{~cm}^{-1}\right)$. Inset shows the liner relationship between SERS intensity of the starred CV peak and CV concentration. (d) Illustration of photocatalysis using magnetic $\mathrm{TiO}_{2}$ NMPs to photo-oxidize CV. The time-dependent plots show the reactivity of a single batch of NMPs throughout repeated photocatalytic cycles. (e) The photo-degradation of $\mathrm{CV}$ in a river water sample using $\mathrm{TiO}_{2}$ NMPs which has been monitored via SERS using single magnetic Au NMPs as the enhancing substrates.

Figure $4 \mathrm{~d}$ shows the photodegradation of $\mathrm{CV}$ in river water using magnetic $\mathrm{TiO}_{2}$ NMPs monitored by UV/vis absorption. In this case the NMPs again have the considerable advantage over colloidal particles that they can be handled as bulk materials and added as a powder to the reaction mixture, they can also be easily separated and recovered after use. Importantly, since the catalytic particle layers are fimly anchored on the surface of the micro-polymer core, the catalytic efficiency of the NMPs showed no decrease even after four 80-minute photocatalysis/particle recovery cycles, despite the prolonged reaction time and extensive handling in between. The data in Figure. 4d were recorded for illustrative purposes using a high $\left(10^{-4} \mathrm{M}\right) \mathrm{CV}$ concentration, which gave a strongly colored purple solution, and with a 
small amount of photoactive $\mathrm{TiO}_{2}$ NMPs (100 mg of NMP for $5 \mathrm{~mL}$ of CV solution). Figure 4e shows a more practical case where the concentration of CV pollutant is $10^{-6}$ M. Using the same amount of magnetic $\mathrm{TiO}_{2} \mathrm{NMPs}$ and $\mathrm{CV}$ solution the dye was degraded to within FDA approved standards in ca. $30 \mathrm{~s}$. This degradation could be conveniently measured using the Au NMP SERS method that was shown in Figure 4c. Based on these experiments, it is anticipated that in practice, $1 \mathrm{~L}$ of river water polluted with $10^{-6} \mathrm{M}$ of $\mathrm{CV}$ can be identified and then purified using only $100 \mathrm{mg}$ of NMP-powder in less than 1.5 hours. Of course, the $\mathrm{TiO}_{2}$ NMPs have general photoactivity towards other organic pollutants. Figure S2, shows the photodegredation of 2-naphthalenethiol monitored by SERS. However, we would stress that all the above were enabled by just 2 variants on the general NMP structure. Therefore, we would anticipate development of numerous other applications based on other combinations of surface NP layers and functional cores.

\section{Conclusion}

In summary, we have described a general and convenient one-pot method to produce robust NMPs, which are micro-powders carrying functional NP layers on the surface. The method combines a promoter induced charge-screening method for forming NP arrays at the liquid-liquid interface with solvent evaporation induced polymer deposition. This approach allows the size and surface morphology of the final micro-particles to be controlled and multigramme amounts to be produced using conventional bench scale apparatus. Importantly, since polymer deposition only occurs at the organic side of the interface, the NP layer in the product is physically exposed and chemically active but at the same time tightly anchored onto the rigid polymer core. Since neither the mechanism for promoter-induced particle selfassembly nor evaporation-induced polymerization deposition involves materialspecific surface functionalization, the method can be generally applied to produce nano-powder materials carrying various different hydrophilic NP surface layers, even including composites. Moreover, functional components such as magnetic or fluorescent materials can be incorporated into the polymer core to add functionality (e.g. magnetization for easy handling, fluorescence for particle tracking) without compromising the nanoscale properties of the surface layer of NPs. Here, we showed that magnetic Au NMPs and $\mathrm{TiO}_{2}$ NMPs could be used in conjunction for rapid, convenient and low cost water monitoring and remediation. However, these are just two of many possible applications since our approach constitutes a simple method for creating tailored NMPs in which selected nanoscale and microscale properties can be rationally combined.

\section{Methods}

Silver nitrate (99.9999\%), gold (III) chloride trihydrate (99.9999\%), chloroplatinic acid hexahydrate, trisodium citrate, tetrabutylammonium nitrate $\left(\mathrm{TBANO}_{3}\right), 2$ naphthalenethiol, crystal violet, methyl orange, 4-mercaptobenzoic acid dichloromethane (DCM), polyvinylpyrrolidone (PVP, M.W. 440,000), 3Mercaptopropionic acid, sodium borohydride, ascorbic acid, benzyl ether, oleic acid, iron (III) acetylacetonate, thioglycolic acid, 2-mercapto-5-benzimidazolesulfonic acid sodium salt dihydrate, 3-mercapto-1-propanesulfonate, 3-mercaptopropionic acid and screw-cap polypropylene centrifuge tubes were purchased from Aldrich Ltd. Polystyrene (M.W. $\approx 100,000)$ was purchased from BDH chemicals Ltd. $50 \mathrm{~nm}$ silica nanospheres $(10 \mathrm{mg} / \mathrm{mL})$ and $20 \mathrm{~nm}$ magnetite nanoparticles $(20 \mathrm{mg} / \mathrm{mL})$ were purchased from nanoComposix. $\mathrm{TiO}_{2}$ colloid $\left(8 \times 10^{-3} \mathrm{~mol} / \mathrm{mL}\right)$ was a kind gift from 
Professor A. Mills. All chemicals were used without further purification. Water used throughout all experiments was low total organic content $(<3.0 \mathrm{ppb}) 18.2 \mathrm{M} \Omega \mathrm{cm}$ water. River water was collected from the river Lagan in Belfast, N.I.

Instrumentation: SEM used a Quanta FEG 250 at an acceleration voltage of $20 \mathrm{kV}$ with standard SEM copper tape or carbon tape as background. SEM images of gold, silver, and platinum NMPs were taken under high chamber vacuum $\left(8 \times 10^{-5}\right.$ mbar $)$ while images of oxide NMPs were taken under low chamber vacuum ( 0.9 mbar). Zeta potential measurements were carried out on a Zetasizer Nano ZS (Malvern Instruments Ltd., U.K.). All the optical images were taken using a SMZ800 Zoom Stereomicroscope (Nikon Instruments Europe B.V. United Kingdom). Particle concentrations were determined using a Nanosight NS300 (Malvern Instruments Ltd., U.K.). SERS spectra were collected with a Perkin Elmer RamanMicro 200 Raman Microscope equipped with a $785 \mathrm{~nm}$ diode laser $(60 \mu \mathrm{m}$ spot diameter at sample) using a total accumulation time of $20 \mathrm{~s}$ and $200 \mathrm{~mW}$ laser power.

Colloid preparation: Citrate-reduced silver colloid was prepared via the Lee and Meisel method [36]. $30 \mathrm{~nm}$ citrate-reduced gold colloid was prepared via TurkevichFrens method with slight modifications [37]. Briefly, $50 \mathrm{mg}$ gold (III) chloride trihydrate dissolved in $50 \mathrm{~mL}$ of DDI water in a round bottom flask was heated to boiling at reflux under vigorous stirring. Then 8 (5.6) $\mathrm{mL}$ of aqueous sodium citrate (1 wt $\%$ ) solution was added into the reaction mixture all at once, which gives $25 \mathrm{~nm}$ gold nanoparticles and $50 \mathrm{~nm}$ gold nanoparticles, respectively. The solution was allowed to react for $30 \mathrm{~min}$ before being cooled down to room temperature. Oleic acid capped magnetic particles were prepared by the thermal decomposition method described in literature and dispersed in $10 \mathrm{~mL}$ of DCM [38]. Pt colloid was prepared following literature methods [39]. The final concentration of $\mathrm{TiO}_{2}, \mathrm{SiO}_{2}$ and commercial magnetite colloid was $3.5 \times 10^{11}$ particles $/ \mathrm{mL}, 1.7 \times 10^{11}$ particles $/ \mathrm{mL}$ and $9.1 \times 10^{11}$ particles $/ \mathrm{mL}$, respectively. The concentration of citrate-reduced gold, silver and platinum colloid was $2 \times 10^{12}, 5.8 \times 10^{11}$ and $1.2 \times 10^{12}$ particles $/ \mathrm{mL}$, respectively.

NMP fabrication: Au and Ag NMPs were prepared by vigorously shaking $5 \mathrm{~mL}$ of aqueous colloid with $1 \mathrm{~mL}$ of polystyrene/DCM solution, $100 \mu \mathrm{L}$ of capping agent and $100 \mu \mathrm{L}$ of $10^{-3} \mathrm{M} \mathrm{TBA}^{+}$for 1-3 $\mathrm{min}$ (Au/Ag hybrid NMPs were prepared by using $3 \mathrm{~mL}$ of $\mathrm{Au}$ colloid and $2 \mathrm{~mL}$ of $\mathrm{Ag}$ colloid, respectively). Commercial magnetite, $\mathrm{TiO}_{2}$, and $\mathrm{SiO}_{2} \mathrm{NMPs}$ were prepared using the same ratio except that no additional capping agent was required. Platinum NMPs were prepared at a different colloid and polystyrene/DCM solution ratio (10:1) in the presence of $100 \mu \mathrm{L}$ of capping agent (PVP) and $50 \mu \mathrm{L}$ of $\mathrm{TBA}^{+}\left(10^{-3} \mathrm{~mol} / \mathrm{L}\right)$. Magnetic encapsulated $\mathrm{TiO}_{2}$ NMPs were prepared by mixing $1 \mathrm{~mL}$ of oleic acid capped magnetic particles, $9 \mathrm{~mL}$ of polystyrene/DCM solution $(0.08 \mathrm{~g} / \mathrm{mL})$ and $40 \mathrm{~mL}$ of $\mathrm{TiO}_{2}$ colloid. The agitated mixture was poured immediately into a polypropylene bowl and subsequently formed emulsions which were left to evaporate for several days at room temperature to form NMPs.

Functionalization of Au NMPs with 4-mercaptobenzoic acid (MBA): $50 \mathrm{~nm}$ PVP capped $\mathrm{Au}$ nanoparticles were used for preparing Au NMPs. MBA was first introduced as an internal standard by mixing $0.5 \mathrm{~mL}$ of $10 \mu \mathrm{M}$ MBA solution with 20 
mg of Au NMPs for 1 min. Au NMPs were then washed with DDI for 5 times and dried in the oven for further use.

Degradation of organic pollutants catalyzed by magnetic $\mathrm{TiO}_{2}$ NMPs: (1) Crystal violet $(\mathrm{CV})$ degradation in river water: In a typical experiment, $100 \mathrm{mg}$ of as-prepared magnetic $\mathrm{TiO}_{2}$ NMPs were dispersed in $5 \mathrm{~mL}$ of $1 \times 10^{-5} \mathrm{M} \mathrm{CV}$ solution and continuously stirred under UV irradiation. A UV-LED (LedEngin Inc.) mounted onto a $50 \times 20 \mathrm{~mm}$ heatsink (Intelligent LED solutions) was used, which had a peak wavelength of $365 \mathrm{~nm}$. The LED was operated with a forward voltage (VF) of 3.5 $\mathrm{dcV}$ and a forward current (IF) of $0.3 \mathrm{~A}$, which gave an overall power of $1.05 \mathrm{~W}$. For UV monitoring samples $(1 \mathrm{~mL})$ were removed and analysed using a Carry 300 scanning UV visible spectrophotometer with a scan rate of $400 \mathrm{~nm} / \mathrm{min}$ in the range 200-800 nm. For stability measurements the NMPs were collected every 80 mins by a neodymium magnet washed with DDI before being re-suspended in a fresh dye solution and irradiated for another $80 \mathrm{~min}$ cycle. 2-naphthalenethiol degradation experiments were carried out using the same procedures starting with $5 \mathrm{~mL}$ of $1 \times 10^{-5}$ M 2-naphthalenethiol solution.

SERS analysis carried out on MBA functionalized Au NMPs: During photocatalytic degradation of organic pollutant (CV and 2-naphthalenethiol), $1 \mathrm{~mL}$ of samples were taken at each time interval into which a single MBA functionalized Au NMP was added. The mixture was then brought to sonication for 1 minute to allow analyte absorption. The Au NMP were extracted and washed with DDI for three times before being used for SERS analysis. All spectra shown were averaged from 5 spectra unless otherwise stated.

\section{References}

1. H. Zhao, S. Sen, T. Udayabhaskararao, M. Sawczyk, K. Kučanda, D. Manna, P. K. Kundu, J.-W. Lee, P. Král, R. Klajn, Reversible trapping and reaction accelerated within dynamically self-assembled nanoflasks, Nature Nanotech. 11 (2016) 82-88.

2. S. M. Taheri, M. Michaelis, T. Friedrich, B. Förster, M. Drechsler, F. M. Römer, P. Bösecke, T. Narayanan, B. Weber, I. Rehberg, S. Rosenfeldt, S. Förster, Self-assembly of smallest magnetic particles, Proc. Natl. Acad. Sci. USA 112 (2015) 14484-14489.

3. Z. Nie, A. Petukhova, E. Kumacheva, Properties and emerging applications of self-assembled structures made from inorganic nanoparticles, Nature Nanotech. 5 (2010) 15-25.

4. F. Li, D. P. Josephson, A. Stein, Colloidal assembly: the road from particles to colloidal molecules and crystals, Angew. Chem. Int. Ed. 50 (2011) 360-388.

5. L. Zhang, Q. Fan, X. Sha, P. Zhong, J. Zhang, Y. Yin, C. Gao, Self-assembly of noble metal nanoparticles into sub-100 nm colloidosomes with collective optical and catalytic properties, Chem. Sci. 8 (2017) 6103-6110.

6. G. Chuong, P. Quang, H. K. Lee, I. Y. Phang, X. Y. Ling, Plasmonic colloidosomes as three-dimensional SERS platforms with enhanced surface area for multiphase sub-microliter toxin sensing, Angew. Chem. Int. Ed. 54 (2015) 9691-9695.

7. T. Bolhorst, S. Shahabi, K. Wörz, C. Petters, R. Dringen, M. Mass, K. Rezwan, Bifunctional submicron colloidosomes coassembled from fluorescent and superparamagnetic nanoparticles, Angew. Chem. Int. Ed. 54 (2015) 118-123. 
8. Z. Qian, S. P. Hastings, C. Li, B. Edward, C. K. McGinn, N. Engheta, Z. Fakhraai, S.-J. Park, Rasberry-like metamolecules exhibiting strong magnetic resonaces, ACS Nano 9 (2015) 1263-1270.

9. M. Tagliazucchi, F. Zou, E. A. Weiss, Kinetically controlled self-assembly of latex-microgel core-satellite particles, J. Phys. Chem. Lett. 5 (2014) 27752780.

10. C. E. Snyder, M. Ong, D. Velegol, In-solution assembly of colloidal water, Soft Matter. 5 (2009) 1263-1268.

11. R. Li, X. Yang, G. Li, S. Li, W. Huang, Core-corona polymer composite particles by self-assembled heterocoagulation based on a hydrogen-bonding interaction, Langmuir 22 (2006) 8127-8133.

12. M. Pang, A. J. Caims, Y. Liu, Y. Belmabkhout, H. C. Zeng, M. Eddaoudi, Synthesis and integration of Fe-soc-MOF cubes into colloidosomes via a single-step emulsion-based approach, J. Am. Chem. Soc. 135 (2013) 1023410237.

13. T. Bollhorst, K. Rezwan, M. Maas, Colloidal capsules: nano- and microcapsules with colloidal particle shells, Chem. Soc. Rev. 46 (2017) 20912126.

14. D. Liu, F. Zhou, C. Li, T. Zhang, H. Zhang, W. Cai, Y. Li, Black gold: plasmonic colloidosomes with broadband absorption self-assembled from monodispersed gold nanoparticles by using a reverse emulsion system, Angew. Chem. Int. Ed. 54, 9596-9600 (2015).

15. D. Lee, D. A. Weitz, Double emulsion-templated nanoparticle colloidosomes with selective permeability, Adv. Mater. 20, 3498-3503 (2008).

16. K. L. Thompson, P. Chambon, R. Verber, S. P. Armes, Can polymersomes form colloidosomes? J. Am. Chem. Soc. 134 (2012) 12450-12453.

17. B. Y. Guan, L. Yu, X. W. Lou, Chemically assisted formation of monolayer colloidosomes on functional particles, Adv. Mater. 28 (2016) 9596-9601.

18. O. J. Cayre, P. F. Noble, V. N. Paunov, Fabrication of novel colloidosome microcapsules with gelled aqueous cores, J. Mater. Chem. 14 (2004) 33513355.

19. Y. Li, S. Sha, Z. Wu, C. Yang, T. Ngai, Facile synthesis of gold nanoparticlecoated polystyrene composite particles templated from pickering emulsion, Colloids Surf. A 494 (2016) 116-124.

20. Y. Xu, M. P. Konrad, W. W. Y. Lee, Z. Ye, S. E. J. Bell, A method for promoting assembly of metallic and nonmetallic nanoparticles into interfacial monolayer films, Nano Lett. 16 (2016) 5255-5260.

21. Y. Xu, M. P. Konrad, J. L. Trotter, C. P. McCoy, S. E. J. Bell, Rapid one-pot preparation of large freestanding nanoparticle-polymer films, Small 13 (2016) 1602163.

22. D. T. Wasan, A possible mechanism of stabilization of emulsions by solid particles, J. Colloid Interface Sci. 150 (1992) 589-593.

23. Q. Chen, Z. Ye, C. Li, H. McCabe, J. Kelly, Y. Xu, S. E. J. Bell, Dataset on Constructing Colloidal Nanoparticles into Dry Nano-Micro-Particle (NMP) Powders with Nanoscale Magnetic, Plasmonic and Catalytic Functionalities, Data in Brief, in press.

24. B. Li, F. Jia, Y.-P. Cao, X.-Q. Feng, H. Gao, Surface wrinkling patterns on a core-shell soft sphere, Phys. Rev. Lett. 106 (2011) 234301.

25. S. S. Datta, H. C. Shum, D. A. Weitz, Controlled buckling and crumpling of nanoparticle-coated droplets, Langmuir 26 (2010) 18612-18616. 
26. R. P. Schwarzenbach, B. I. Escher, K. Fenner, T. B. Hofstetter, C. A. Johnson, U. von Gunten, B. Wehrli, The challenge of micropollutants in aquatic systems, Science 313 (2006) 1072-1077.

27. S. D. Richardson, T. A. Ternes, Water analysis: emerging contaminants and current issues, Anal. Chem. 90 (2018) 398-428.

28. A. Alsbaiee, B. J. Smith, L. Xiao, Y. Ling, D. E. Helbling, W. R. Dichtel, Rapid removal of organic micropollutants from water by a porous $\beta$ cyclodextrin polymer, Nature 529 (2016) 190-194.

29. F. Mashkoor, A. Nasar, Inamuddin, A. M. Asiri, Exploring the reusability of synthetically contaminated wastewater containing crystal violet dye using Tectona grandis sawdust as a very low-cost adsorbent, Sci. Rep. 8 (2018) 8314.

30. World Health Organization (WHO) and the United Nations Children's Fund (UNICEF), Progress on drinking water, sanitation and hygiene: 2017 update and SDG baselines. Geneva: 2017. Licence: CC BY-NC-SA 3.0 IGO.

31. D.-W. Li, W.-L. Zhai, Y.-T. Li, Y.-T. Long, Recent progress in surface enhanced Raman spectroscopy for the detection of environmental pollutants, Microchim. Acta 181 (2014) 23-43.

32. P. C. Pinheiro, A. L. Daniel-da-Silva, H. I. S. Nogueira, T. Trindade, Functionalized inorganic nanoparticles for magnetic separation and SERS detection of water pollutants, Eur. J. Inorg. Chem. 30 (2018) 3443-3461.

33. J. Du, J. Xu, Z. Sun, C. Jing, Au nanoparticles grafted on $\mathrm{Fe}_{3} \mathrm{O}_{4}$ as effective SERS substrates for label-free detection of the 16 EPA priority polycyclic aromatic hydrocarbons, Anal. Chim. Acta 915 (2016) 81-89.

34. X. Jiang, M. Yang, Y. Meng, W. Jiang, J. Zhan, Cysteamine-modified silver nanoparticles aggregates for quantitative SERS sensing of pentachlorophenol with a portable Raman spectrometer, ACS Appl. Mater. Interfaces 5 (2013) 6902-6908.

35. L. G. Rushing, E. B. Hansen Jr., Confirmation of malachite green, gentian violet and their leuco analogs in catfish and trout tissue by high-performance liquid chromatography utilizing electrochemistry with ultraviolet-visible diode array detection and fluorescence detection, J. Chromatogr. B 700 (1997) 223231.

36. P. C. Lee, D. Meisel Adsorption and surface-enhanced Raman of Dyes on silver and gold sols, J. Phys. Chem. 86 (1982) 3391-3395.

37. G. Frens, Controlled nucleation for the regulation of the particle size in monodisperse gold suspensions, Nature 241 (1973) 20-22.

38. C. Moya, X. Batlle, A. Labarta, The effect of oleic acid on the synthesis of $\mathrm{Fe}_{3-\mathrm{x}} \mathrm{O}_{4}$ nanoparticles over a wide size range, Phys. Chem. Chem. Phys. 17 (2015) 27373-27379.

39. K. Kim, K. L. Kim, H. B. Lee, K. S. Shin, Surface-enhanced Raman scattering on aggregates of platinum nanoparticles with definite size, J. Phys. Chem. C 114 (2010) 18679-18685.

\section{Acknowledgements}

The authors thank Matthew Pilgrim for help in preparing SEM samples and Prof. Andrew Mills for providing $\mathrm{TiO}_{2}$ colloid. Z. Y. and Y. X. were funded by a University Special Research Scholarship (Q. U. B.). J. K. was funded by the Department of Education (N.I.). H. M. was funded by the Engineering and Physical Sciences Research Council. N. S. was funded under a QUB Pioneer Research Program. 


\section{Competing interests}

The authors declare no competing financial interests in relation to this work.

\section{Materials \& Correspondence}

Any correspondence regarding the work in this manuscript should be addressed to yxu18@qub.ac.uk or S.Bell@qub.ac.uk.

\section{Data Availability}

The raw/processed data required to reproduce these findings cannot be shared at this time as the data also forms part of an ongoing study.

\section{TOC}

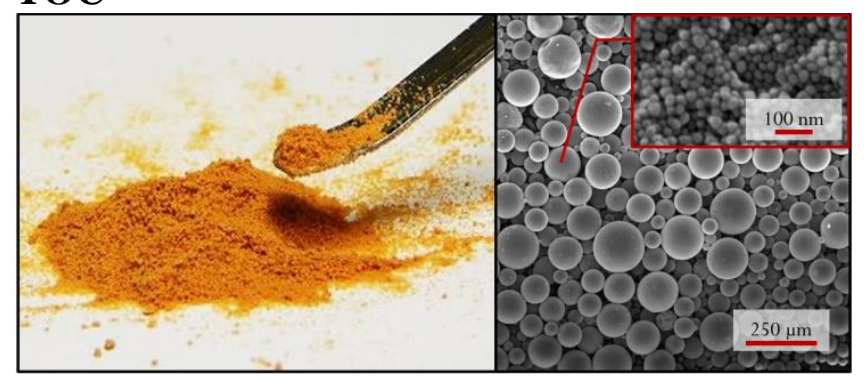

